\title{
MODELING OF $\mathrm{H}_{2} / \mathrm{O}_{2}$ SINGLE-ELEMENT ROCKET THRUST CHAMBER COMBUSTION AT SUB- AND SUPERCRITICAL PRESSURES WITH DIFFERENT COMPUTATIONAL FLUID DYNAMICS TOOLS
}

\author{
H. Riedmann ${ }^{1}$, D. Banuti ${ }^{2}$, B. Ivancic ${ }^{1}$, O. Knab ${ }^{1}$, \\ and K. Hannemann ${ }^{2}$ \\ ${ }^{1}$ Airbus DS GmbH \\ Munich 81663, Germany \\ ${ }^{2}$ German Aerospace Center (DLR) \\ Göttingen 37073, Germany
}

\begin{abstract}
This paper derives from the cooperation between DLR and Airbus DS within the work package "CFD Modeling of Combustion Chamber Processes" conducted in the frame of the Propulsion 2020 project. In a joint strategy, DLR Göttingen and Airbus DS Ottobrunn have identified a number of test cases with gradually growing complexity where adequate test data are available for proper successive validation of the computational fluid dynamics (CFD) tools to be used in an industrial environment. This work highlights the simulation results for the Mascotte A-10 and A-60 test cases as presented at the 2nd International Workshop on Rocket Combustion Modeling in Lampoldshausen 2001 by ONERA and SNECMA [1]. These two test cases are characterized by different chamber pressures (10 and 60 bar) and, consequently, by oxygen injection conditions which are subcritical in one case and transcritical in the other case. The test cases are treated with three different CFD codes: the DLR TAU Code (only A-60 case), the Airbus DS in-house tool Rocflam3, and the commercial CFD tool ANSYS CFX incorporating several modeling extensions by Airbus DS. To the knowledge of the authors, this paper is the first one which covers both the A-10 and the A-60 test cases.
\end{abstract}

\section{INTRODUCTION}

The main target of the cooperation between DLR and Airbus DS within Propulsion 2020 is extending knowledge and competence in the area of rocket propulsion

(C) The Authors, published by EDP Sciences. This is an open access article distributed under the terms of the Creative Commons Attribution License 4.0 (http://creativecommons.org/licenses/by/4.0/). 
combustion devices, particularly, with regard to test, modeling, and simulation capabilities. One of the work packages is focused on CFD modeling of the hot gas flow and the hot gas-side heat transfer in rocket thrust chambers. This work package is executed in close cooperation between the DLR Institute of Aerodynamics and Flow Technology and Airbus DS. The first results emanating from this cooperation have been published by Ivancic et al. [2]. They cover the numerical simulation of the well-known Penn State test case which deals with the combustion of preburned, i. e., gaseous, hydrogen, and oxygen in a single-element combustion chamber under supercritical conditions. Thereby, very good agreement could be achieved between the three applied CFD tools and the available wall heat flux test data. In the second step, these CFD tools are now used for simulating the Mascotte test cases A-10 and A-60 (see [1] for official test case descriptions) where two very similar combustion chambers (see section 2 for details) are operated in the sub- and supercritical pressure regime, respectively. In order to be applicable for the simulation of combustion and flow in real rocket thrust chambers, a simulation tool must be able to cope with these conditions.

The three applied CFD tools are the Airbus DS in-house tool Rocflam3, the commercial code ANSYS CFX (equipped with several modeling extensions implemented by Airbus DS via the User Fortran interface), and the DLR TAU code. Further details on these codes and on the used models are given in section 3 . The main goal of performing analog Reynolds-average Navier-Stokes (RANS) simulations with three different tools is to provide a broad spectrum of data for the assessment of the applied modeling methodologies regarding their suitability for rocket thrust chamber simulations.

In the end, the developed CFD tools with their specific models and methods will be applicable in the design and optimization processes of real rocket thrust chamber hardware. Hence, each tool must comprise a package of models enabling it to simulate multiinjector full-scale combustors in a reasonable time frame thereby encompassing the driving mechanisms such as propellant disintegration, combustion, and heat transfer. Performing single-element combustor simulations as addressed in this paper is considered a valuable milestone for the validation of the CFD tools. Thereby, it should always be kept in mind that the elaborated settings must be transferable to multiinjector simulations since only the latter can support engine layout and contribute to reduce development time and cost (see Knab et al. [3]).

\section{TEST CASE DESCRIPTIONS}

\subsection{Mascotte A-10 Test Case}

The Mascotte A-10 test case has been described in detail by Vingert and Habiballah [4]. Therefore, only a short description is given here. In this case, the Mas- 


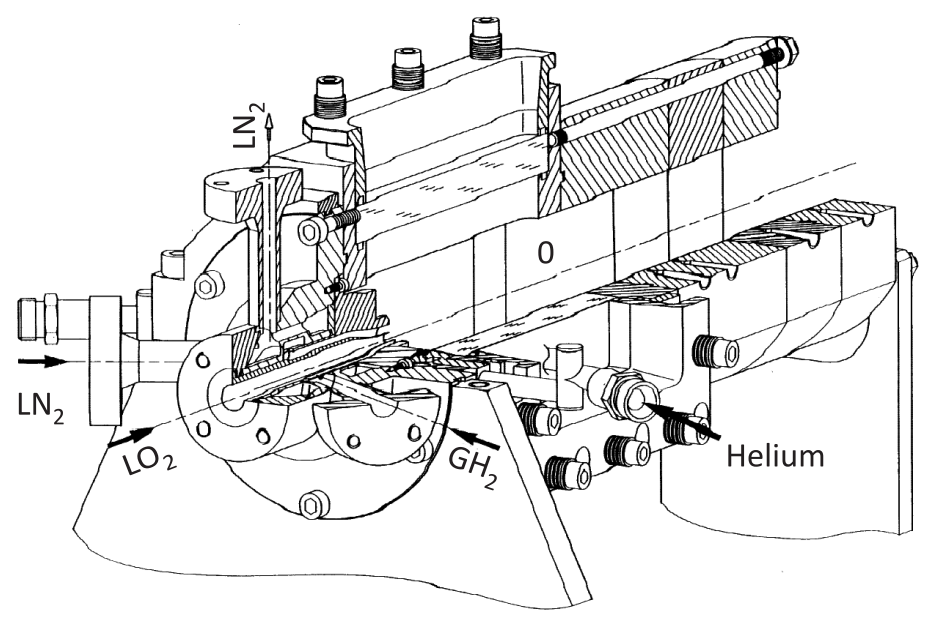

Figure 1 Sketch of the Mascotte combustor (taken from [4])

cotte $\mathrm{H}_{2} / \mathrm{O}_{2}$ combustor works at a subcritical chamber pressure of 10 bar and a mixture ratio of 2.11. The chamber has one shear-coaxial injector located on the center axis. Liquid oxygen ( $\mathrm{LOx}$ ) is injected in the core of the injector with a temperature of $85 \mathrm{~K}$ whereas the surrounding hydrogen is injected in gaseous state at $287 \mathrm{~K}$. Liquid nitrogen is used to cool down the oxygen before injection. The oxygen mass flow rate is $50 \mathrm{~g} / \mathrm{s}$ and the hydrogen mass flow rate is $23.7 \mathrm{~g} / \mathrm{s}$. The diameter of the oxygen tube is $5 \mathrm{~mm}$ and the diameters of the hydrogen annulus are 5.6 and $12 \mathrm{~mm}$. This results in a momentum ratio of $J=15.4$ and a velocity ratio of 146.3 between fuel and oxidizer. A sketch of the Mascotte combustor is shown in Fig. 1. The chamber has a square cross section with an edge length of $50 \mathrm{~mm}$. The distance between the injection plane and the throat is $478 \mathrm{~mm}$. The throat diameter is $15 \mathrm{~mm}$. For the purpose of optical diagnostics, the chamber is equipped with helium-cooled quartz windows on two sides. The helium cooling mass flow rate is not given in the test case description and, consequently, is not taken into account by the simulations. Nevertheless, one must be aware of the nonquantified inaccuracy which is introduced due to this simplification when comparing simulation and experiment.

Furthermore, it is noticeable that neither the operation point (combination of chamber pressure and mixture fraction) of the Mascotte A-10 test case nor the geometry of the chamber is representative for common rocket engines. The area contraction ratio between chamber and throat is $\varepsilon_{c}=14.1$ and the characteristic chamber length (quotient of chamber volume and throat area) is $l^{*}=V_{c} / A_{t}$ 


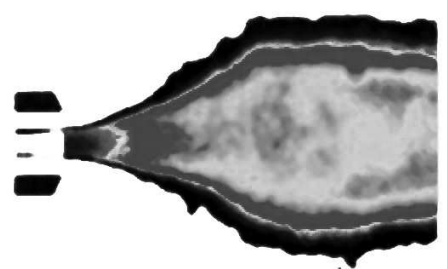

(a)

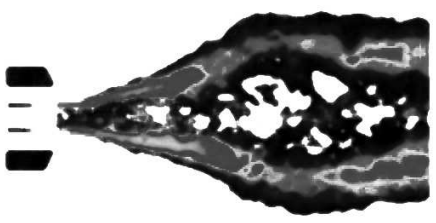

(b)

Figure 2 Mascotte A-10: $(a)$ average $\mathrm{OH}^{*}$ emission image; and (b) Abel-transformed emission image (taken from [5])

$=6.6 \mathrm{~m}$. Both values are notably higher than they are for common rocket engines like, for example, the space shuttle main engine, Vulcain, and HM7. For those engines, contraction ratios of the order of $2.5 \leq \varepsilon_{c} \leq 3$ and characteristic lengths of $0.7 \leq l^{*} \leq 0.8 \mathrm{~m}$ can be found. Nevertheless, the A-10 test case is a very interesting and well reported test case to investigate the combustion of cryogenic propellants which is the reason why it has been chosen for this work.

Several authors have published experimental data resulting from the Mascotte A-10 case. The experimental data which are used for comparison with the numerical results in this work originate from two publications: there are an Abel-transformed emission image and mean temperature profiles published by Candel et al. [5] as well as additional mean temperature profiles published by Grisch et al. [6]. The emission images are shown in Fig. 2.

Figure $2 a$ shows the average emission image whereas Fig. $2 b$ shows the Abeltransformed emission image which is suited to be compared with contour plots of the $\mathrm{OH}^{*}$ radiation computed from numerical simulations. Note that the Abel transformation acts on the assumption of an axisymmetric flame shape which is not necessarily applicable for the Mascotte combustor that has a square cross section. For the available CFD results, the evaluation of the $\mathrm{OH}^{*}$ radiation is performed according to the a posteriori method proposed by Fiala and Sattelmayer [7]. The aforementioned temperature measurements have been obtained by coherent anti-Stokes Raman scattering (CARS) thermometry. The mean temperature values, the measurement locations, the standard deviations, and the validation rates can be found in the cited publications $[5,6]$. While Candel et al. [5] only show test data based on $\mathrm{H}_{2}$ concentrations, Grisch et al. [6] also show test data based on $\mathrm{H}_{2} \mathrm{O}$ concentrations. Both are used for comparison with the simulation results (see subsection 4.1). Although being highly turbulent, the combustion process is said to be stationary in both cited publications which is a main requirement for the RANS simulations presented in this work. 


\subsection{Mascotte A-60 Test Case}

The Mascotte A-60 test case has been described in detail by Thomas and Zurbach [8]. A short description highlighting the differences to the A-10 test case is given here. In this case, the Mascotte combustor works at a chamber pressure of 60 bar (supercritical for both $\mathrm{H}_{2}$ and $\mathrm{O}_{2}$ ) and a mixture ratio of 1.4. While the chamber pressure is representative for common rocket thrust chambers, the mixture ratio is not. The injection temperatures are the same as for the A-10 test case $\left(85 \mathrm{~K}\right.$ for $\mathrm{O}_{2}$ and $287 \mathrm{~K}$ for $\mathrm{H}_{2}$, i. e., $\mathrm{O}_{2}$ is injected transcritical and $\mathrm{H}_{2}$ supercritical), but the propellant mass flow rates are higher: the oxygen mass flow rate is $100 \mathrm{~g} / \mathrm{s}$ and the hydrogen mass flow rate is $70 \mathrm{~g} / \mathrm{s}$. The diameter of the oxygen tube is again $5 \mathrm{~mm}$ but the diameters of the hydrogen annulus are 5.6 and $10 \mathrm{~mm}$, i. e., the outer diameter of the hydrogen annulus is reduced by $2 \mathrm{~mm}$ compared to the A-10 test case. This results in a momentum ratio of $J=13.8$ and a velocity ratio of 54.3 between fuel and oxidizer, i. e., while the momentum ratio is comparable to A-10, the velocity ratio is significantly lower. The throat diameter is $6 \mathrm{~mm}$ smaller than for the A-10 case and thus amounts to $9 \mathrm{~mm}$.

Figure 3 shows the $\mathrm{OH}^{*}$ emission images for the A-60 test case. These pictures have been obtained from [9]. To the knowledge of the authors, no discrete temperature values from CARS measurements are available in literature for the A-60 load point exactly as it has been defined by Thomas and Zurbach [8]. However, there are CARS data available from Habiballah et al. [10] who refer to the A-60 load point as an operating point with a hydrogen mass flow rate of $75 \mathrm{~g} / \mathrm{s}$

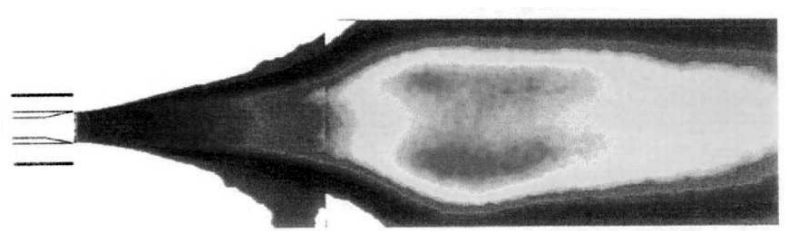

(a)

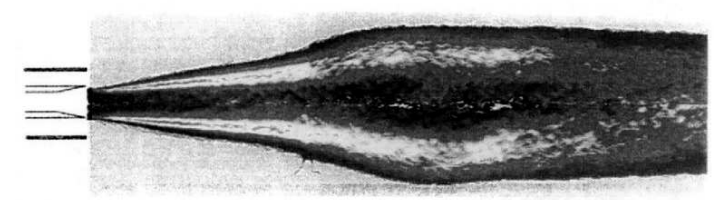

(b)

Figure 3 Mascotte A-60: (a) average $\mathrm{OH}^{*}$ emission image; and (b) Abel-transformed emission image (taken from [9]) 
which is $5 \mathrm{~g} / \mathrm{s}$ more than Thomas and Zurbach [8] state for A-60. Nevertheless, these data will be used for comparison with the simulation results in subsection 4.2 whereby it has to be kept in mind that the load points from simulation and experiment not being identical introduces a further unknown deviation when comparing one with the other. An axial temperature distribution for the A-60 test case has been published by Poschner and Pfitzner [11] which is also used for comparison. Because the authors neither give detailed information about the origin of these data nor about the radial location where the values have been obtained, they are here interpreted as temperature values on the axis.

\section{APPLIED TOOLS, MODELS, AND SETTINGS}

\subsection{Rocflam3}

The three-dimensional (3D) CFD tool Rocflam3 is currently under development at Airbus DS in Ottobrunn as designated successor of the two-dimensional (2D) / axisymmetric in-house code Rocflam-II. The continuous gas phase is treated using an Euler description solving the Favre-averaged conservation equations for mass, momentum, and enthalpy in three spatial dimensions. The equations are discretized with a finite-volume scheme for nonorthogonal, boundaryfitted, and block-structured grids according to the semiimplicit method for pressure linked equations (SIMPLE) algorithm described by Patankar and Spalding $[12]$.

The turbulence model used in this work is the standard $k-\epsilon$ model by Jones and Launder [13]. In order to account for the interaction between turbulence and chemistry, an equilibrium-based presumed probability density function (PDF) approach is applied. Thereby, the thermodynamic and transport properties obtained for chemical equilibrium are superposed with a one-dimensional (1D) Beta-PDF for the mixture fraction and then integrated over the mixture fraction range. Therefore, additional transport equations are solved for the mixture fraction and its variance. The computed values for enthalpy, pressure, mixture fraction, and mixture fraction variance are used for a table lookup of the integrated quantities. These quantities are temperature, density, molar mass of the mixture, heat conductivity, specific heat capacity, viscosity, species concentrations, and the derivative of pressure with respect to density at constant pressure which is used in the SIMPLE algorithm for the compressible Navier-Stokes equations.

Rocflam3 comprises several approaches to model the propellant injection. The most common one is the inlet boundary with prescribed mass flow rate. This boundary condition is well suited for the injection of gaseous and supercritical propellants where a continuum exists. It has been chosen for the A-60 test case. 
When it comes to the injection of liquid propellants, the propellants are assumed to form a spray of small droplets which deserves a special treatment. Therefore, the injection of liquid propellants can be modeled via a Lagrangian module for propellant droplet tracking and evaporation in Rocflam3. This model has been adopted from Rocflam-II. A detailed description can be found in the work by Kniesner et al. [14]. It has been chosen for the A-10 test case.

The Lagrangian module is loosely coupled to the Euler module. Propellants injected in liquid state are tracked throughout the flow field until they have vaporized. Thereby, mass, momentum, and enthalpy are released and are transferred to the gas phase via source terms in the conservation equations.

To keep the computational effort low, not every single droplet of the injected propellant is tracked but only a certain number of representative trajectories are computed. Therefore, discrete diameters and injection angles are defined and assigned with specific probabilities. For the propellant droplet tracking procedure, an equation of motion must be solved for each of these representative trajectories. Droplet vaporization is approximated using the model by Abramzon and Sirignano [15].

For the A-10 test case, Vingert and Habiballah [4] propose the injection of the oxidizer droplets on a conical solid boundary resolved by the grid to represent the LOx core. This has not been done with Rocflam3 in order to keep the computational grid as simple as possible. The same methodology is also applied for multiinjector configurations (see [16]) which are in the main focus of Rocflam3. As proposed by Vingert and Habiballah [4], the oxygen droplet size distribution is modeled using a Rosin-Rammler distribution with a mean diameter of $130 \mu \mathrm{m}$. In the simulation, this distribution is approximated using 25 discrete droplet sizes. The injection velocity is $2.18 \mathrm{~m} / \mathrm{s}$ as given by Vingert and Habiballah [4]. The droplets enter the domain along 30 discrete vectors with off-axis angles between $0^{\circ}$ and $15^{\circ}$. With these settings, the spray is modeled by 23,500 representative trajectories. The gaseous hydrogen is brought into the domain not via an inlet boundary but via source terms for mass, momentum, and enthalpy.

Figure 4 shows the numerical grid used for the 3D simulation of the A-10 test case. Both the faceplate and the liner walls are modeled as adiabatic no-slip walls. Symmetry boundary conditions are applied in the $x-z$ - and the $x-y$ planes. A supersonic boundary condition is set on the outlet. The grid consists of a total number of about $1.6 \cdot 10^{6}$ grid cells. The grid which has been used for the $2 \mathrm{D}$ /axisymmetric computations has similar resolutions in both axial and radial directions. In order for the chamber cross section in the $2 \mathrm{D} /$ axisymmetric simulation to be equal to the real value, a diameter of $56.42 \mathrm{~mm}$ has been chosen.

Almost the same grid (only the throat diameter is different) is used for the A-60 test case. However, as mentioned earlier, the Lagrangian module is not applied in this case. Several studies have been conducted with Rocflam3 using the 


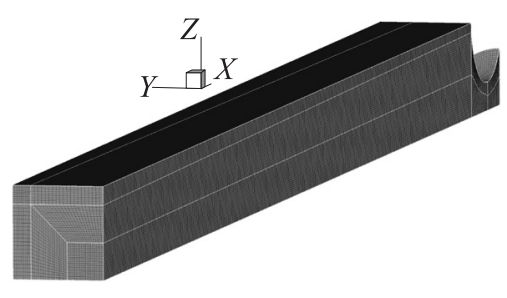

(a)

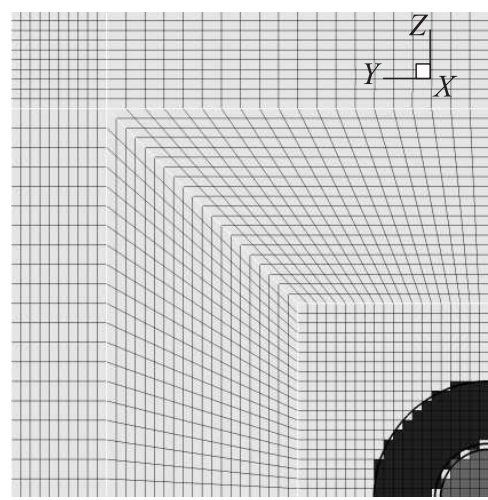

Hydrogen

Oxygen

$(b)$

Figure 4 Rocflam3 grid for the Mascotte A-10 test case: (a) 3D view; and (b) faceplate

Lagrangian module for the injection of the transcritical oxygen in the A-60 test case, but while they all showed good convergence behavior and plausible results, the agreement with the characteristic flame shape known from the experimental data (see Fig. 3) was not satisfactory. Instead, a mass flow inlet boundary condition is prescribed for both fuel and oxidizer, i. e., a so-called dense gas approach is applied. For the simulations with this inlet boundary condition, the prescribed inflow turbulence intensity has shown to have a great influence on the flame shape: increasing the turbulence intensity leads to a shortening of the recirculation zone which develops between the injection element and the chamber wall and, thereby, moves the position where the flame expands in upstream direction.

\subsection{DLR TAU Code}

The DLR TAU code is a hybrid (structured/unstructured) grid finite-volume flow solver for the compressible Euler and Navier-Stokes equations. Turbulence models ranging from RANS one- and two-equation models to detached and large eddy simulations are available in TAU. It has been validated for a range of steady and unsteady flow cases $[17,18]$ including combustion in a rocket preburner [2].

For the current work, a MAPS+ Riemann solver is used in a Godunov type framework to handle low Mach numbers and high density gradients. Spatial second order is reached by a MUSCL (monotonic upwind scheme for conservation laws) reconstruction. Stabilization, if necessary for the computation of high den- 
sity ratio shear layers, is carried out by reducing the spatial order to 1.95 . In this way, the numerical damping is minimally increased while mass, momentum, and energy remain conserved. Turbulence is accounted for using the one-equation Spalart-Allmaras [19] model. Chemical source terms are computed using Arrhenius reaction rates. Here, Jachimowski's [20] mechanism is used. It involves 8 species $\left(\mathrm{H}_{2}, \mathrm{OH}, \mathrm{H}_{2} \mathrm{O}, \mathrm{H}_{2} \mathrm{O}_{2}, \mathrm{O}, \mathrm{O}_{2}, \mathrm{H}\right.$, and $\left.\mathrm{HO}_{2}\right)$ and 17 reactions. Analyzing computations of a gas-gas combustor, Lempke et al. [21] found practically no difference in the temperature field when comparing laminar chemistry to a PDF model of turbulent combustion. Thus, while envisioned for future studies, no dedicated turbulence-chemistry interaction model is employed here.

Cryogenic oxygen, as encountered in rocket engines, thermodynamically acts as a real gas. Following an Eulerian rather than Lagrangian approach to injection modeling, a new real gas mixture model has been developed and implemented in the DLR TAU code, which only treats oxygen as a real gas and all other species as ideal. This is consistent with physical findings [22,23]. Cryogenic oxygen is treated as an Eulerian continuum. This is straightforward for A-60 where due to nonexistent latent heat and surface tension, droplets are not observed [24]. Oxygen real gas properties are computed from the high fidelity modified BenedictWebb-Rubin equation of state (EoS) of Younglove [25] and stored in a library during a preprocessing step. Thermodynamic state variables, such as pressure, enthalpy, heat capacities, speed of sound, etc., are all computed consistently from the real gas EoS. Real gas corrections to the transport coefficients are evaluated following Lemmon and Jacobsen [26]. For all other species, an ideal gas EoS is solved. The model is described in more depth in [27-29]. It has been verified and validated by fluid data comparison with NIST, zaro-dimensional vaporization of LOx, and $1 \mathrm{D}$ combustion.

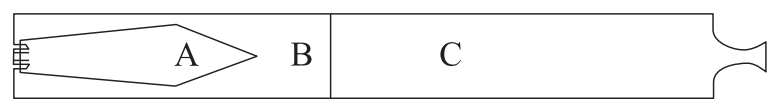

Figure 5 TAU grid structure

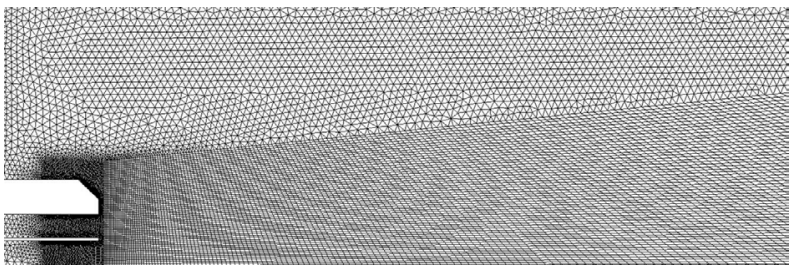

Figure 6 Close up of injector region with structured subgrid and unstructured surrounding grid for TAU 
As the injection model is Eulerian, boundary conditions simply correspond to the physical inflow conditions measured in the experiment. The numerical setup for the A-60 test case is illustrated in Figs. 5 and 6 . The flow field is modeled as axisymmetric with a cross section corresponding to the physical chamber, i.e., the model chamber diameter is $56.42 \mathrm{~mm}$. The inflow is treated with respective Dirichlet boundary conditions at the injector. Figure 5 shows the grid structure: the shear layer and the dense LOx core are resolved and enclosed in a structured subgrid A which is embedded in an unstructured grid B. A close up of this region can be seen in Fig. 6. Region $\mathrm{C}$ is a coarser unstructured grid in a flow region where resolution requirements are relaxed. All walls are considered as inviscid Euler walls. The pressure is determined in the simulation by the flow through the choked nozzle and not prescribed as a boundary condition.

The corresponding version of the present article included in the proceedings of 6th EUCASS conference includes preliminary results for the A-10 test case obtained with the aforementioned attempt. While we feel that these results represent an adequate contribution to a conference, the utilized approach requires a more detailed evaluation before it should be included in an archival publication. Therefore, the corresponding results are not included in the present article.

\subsection{ANSYS CFX}

The commercial ANSYS CFX software is a high-performance, general purpose 3D fluid dynamics program that has been applied to solve wide-ranging fluid flow problems for over 20 years. The heart of ANSYS CFX is its advanced solver technology. The highly parallelized solver is the foundation for an abundant choice of physical models to capture many types of phenomena related to fluid flow. Due to the fact that the model development of ANSYS CFX is focused on turbomachinery issues, important physical problems related to rocket combustors cannot be simulated with CFX directly. A customization of CFX is essential for the successful usage in rocket combustion simulations. Therefore, high effort has been invested in the adaptation of the CFX solver for the usage under these extreme thermodynamic conditions. This means that some in-house model extensions and adaptations are necessary to achieve results with an acceptable quality. The modifications are attached to CFX, e.g., by the User FORTRAN Interface. The adaption process and the status of the Airbus DS tool development are shown in [2].

Apart from the investigation of the influence of the different turbulence models and turbulence intensities, the sensitivity of the combustion modeling was investigated. CFX can use the Flamelet model, eddy dissipation concept, and an equilibrium-based turbulent combustion model for the simulation of rocket combustion chambers. The equilibrium-based combustion model is not a CFX 
standard combustion model. It was developed and implemented via user defined functions into CFX by Airbus DS.

As for the chemical equilibrium model, only two transport equations have to be solved for the CFX-Flamelet approach which leads to very low computational costs. The species composition for a certain mixture fraction is read from Flamelet tables which are generated in a preprocessing step. Unlike the equilibrium combustion model, the CFX-Flamelet approach also includes chemical nonequilibrium conditions characterized by the scalar dissipation rate. For high scalar dissipation rates or shear strain, the Flamelet model accounts also for possible (local) extinction of the flame. The influence of turbulence on combustion is considered via presumed PDFs. A deficiency of the current implementation of the Flamelet model is that the chemistry table only depends on the mixture fraction, mixture fraction variance, and scalar dissipation rate, but not on the enthalpy (for constant mixture fraction) or pressure. This means that, strictly speaking, the species composition is only correct for constant pressure and adiabatic conditions. This is more or less accurate enough in the flame region, but close to strongly cooled walls or in nozzle extensions where the pressure drops, the inaccuracies are rising.

The fluid properties in CFX are extended with real gas data and sophisticated mixing rules for viscosity and heat conductivity are implemented also via user defined functions.

The turbulence model in the CFX-simulations is the Shear Stress Transport (SST) model [28]. It is a $k-\omega$-based turbulence model, which has advantages for the near-wall treatment in the low Reynolds number region. In this region, the $k-\omega$-based turbulence model is more accurate and robust compared to the $k-\varepsilon$ turbulence model. In the free shear flow region, the SST model switches with a blending function from the $k-\omega$ to the $k-\varepsilon$ turbulence model which has here its strength.

For both Mascotte cases (A-10 and A-60, i. e., sub- and supercritical pressure regimes of a cryogenic $\mathrm{LOx} / \mathrm{H}_{2}$ injection), a two-phase flow (particle-laden gas flow) with subsequent spray combustion is an appropriate model description. In CFX, the two-phase flow is simulated via a Lagrange approach for the droplets and an Euler description for the gas phase. The evaporating LOx droplets are accounted in the gas solution via source terms for mass, momentum, mixture fraction, and energy. When approaching the critical point, the two-phase regime transforms to a single-phase description. As shown in earlier publications (see, e.g., [31]), this regime can still be treated by a Lagrangian approach, taking special care of the fact that surface tension and evaporation enthalpy vanish above the critical point.

In this Lagrangian approach, injection velocities, densities, and temperatures of the sub- and transcritical propellants are captured correctly. The hydrogen jet is completely described within the Euler equations (dense gas approach) and appropriate fluid property data bases are essential for accurate description of the 


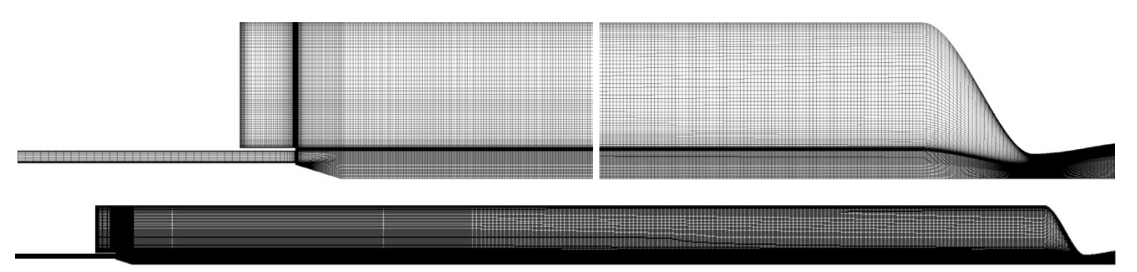

Figure 7 Numerical mesh for the CFX simulations of the Mascotte A-10 test case

mixing process of the cryogenic propellants (LOx droplets and dense hydrogen) with the surrounding hot gases. The thermodynamic and transport properties of the propellants are stored in real gas property tables down to cryogenic temperatures. For lower temperatures, i. e., below $700 \mathrm{~K}$, these tables are generated with a fluid property data base to correctly capture real gas effects. For higher temperatures, i. e., above $1000 \mathrm{~K}$, ideal gas properties can be assumed. For the transition range between 700 and $1000 \mathrm{~K}$, a blending function is implemented ensuring a smooth transition between both data sets (real to ideal gas properties). By this approach, it is ensured that also the subsequent combustion process at much higher temperatures is covered accurately. Moreover, validation studies revealed that the mixing laws are important in order to get satisfying results. Especially for a correct simulation of the mixing process and wall heat fluxes, a proper modeling of the gas mixture properties (like thermal conductivity) is essential.

The CFX simulations shown here are quasi-2D/axisymmetric simulations assuming rotation symmetry of the geometry. The model chamber diameter is $56.42 \mathrm{~mm}$, i. e., the cross section is equal to the original value. The LOx droplet injection is realized via a cone at the $\mathrm{LOx}$ inlet representing the $\mathrm{LOx}$ core, where the droplets are released with correct mass flow, momentum, and energy but with assumed droplet size distributions and velocity angles - just as proposed by Vingert and Habiballah [4] for the A-10 test case. The same approach is applied for the A-60 test case, but the droplet size distribution mean diameter is reduced to $80 \mu \mathrm{m}$. Figure 7 shows the numerical mesh for the 2D/axisymmetric CFX simulations.

\section{EVALUATION AND COMPARISON OF THE SIMULATION RESULTS}

\subsection{Mascotte A-10 Test Case}

Before comparing the simulation results from Rocflam3 and CFX, attention will be attracted to the temperature fields computed by Rocflam3 in a 3D and 


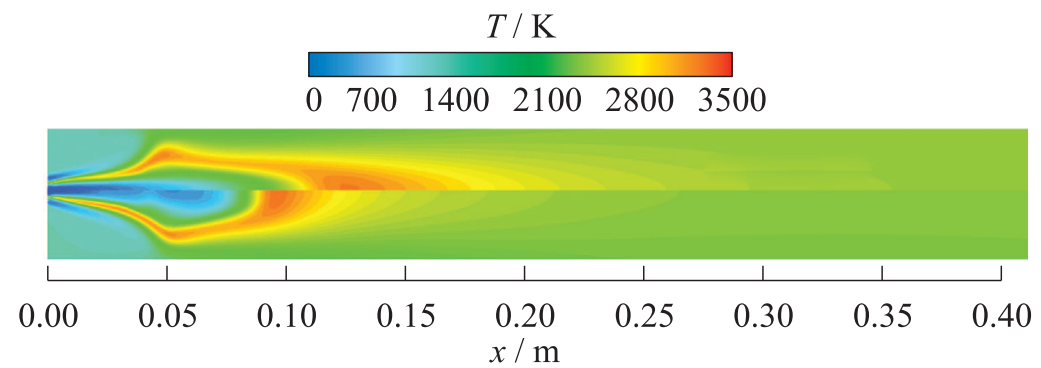

Figure 8 Temperature fields for the Mascotte A-10 test case - Rocflam3 3D (upper part) vs. $2 \mathrm{D} /$ axisymmetric (lower part)

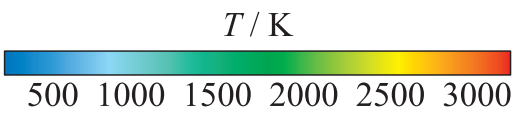

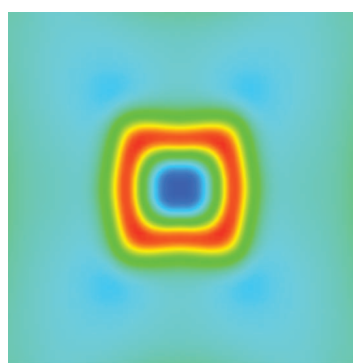

(a)

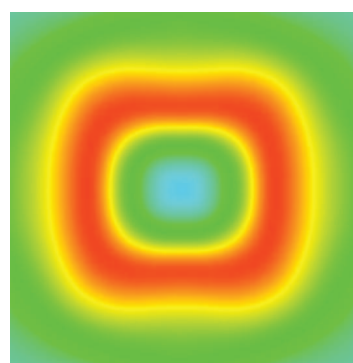

(b)

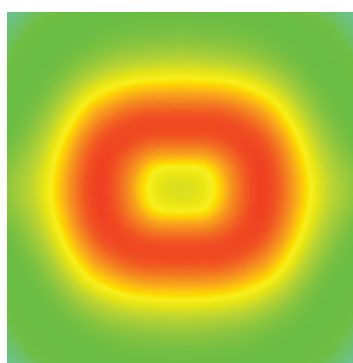

(c)

Figure 9 Temperature field for the Mascotte A-10 test case - Rocflam3 slices at distinct axial locations: $(a) x=0.033 \mathrm{~m}$; (b) 0.067 ; and $(c) x=0.1 \mathrm{~m}$

a 2D/axisymmetric simulation visualized in Fig. 8. While the results look quite similar in the first $0.05 \mathrm{~m}$, they deviate from one another further downstream. The flame is much longer in the $3 \mathrm{D}$ case. In addition to that, the $3 \mathrm{D}$ simulation reveals that the quadratic chamber geometry has a significant influence on the flame shape via the recirculation zones in the four corners. This is visualized in Fig. 9 where the temperature fields for three slices at distinct axial locations are shown for the Rocflam3 simulation. (Note that $360^{\circ}$ are shown here while the actual Rocflam 3 domain covers only $90^{\circ}$.) This result shows that it is worthwhile to make some efforts towards the 3D simulation capability even for this simple single element configuration. Therefore, all following figures show Rocflam3 3D results. 


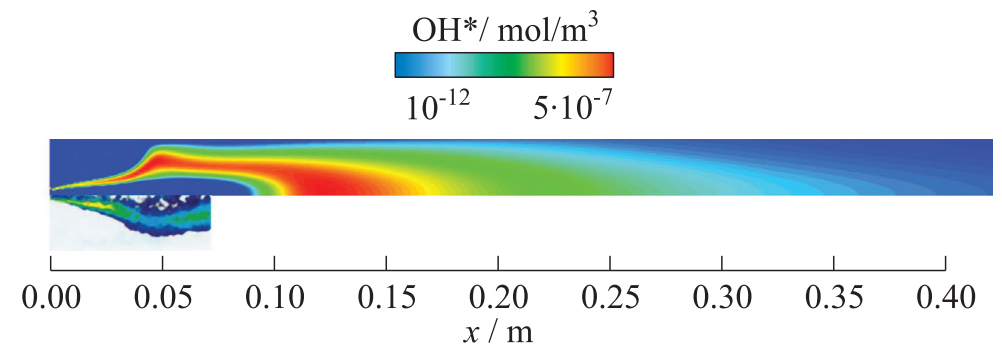

(a)

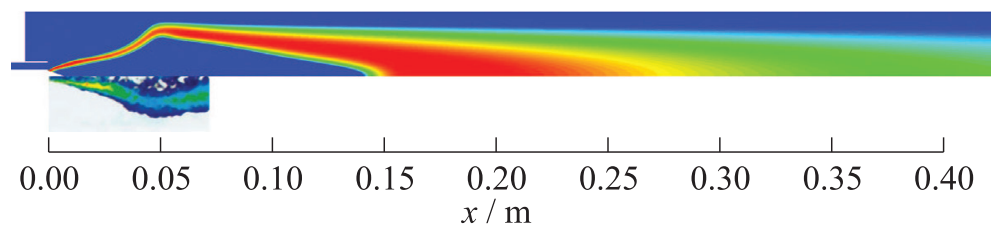

(b)

Figure $10 \mathrm{OH}^{*}$ radiation fields for the Mascotte A-10 test case (comparison between simulation results and emission image from test [5], exponential color scale): (a) Rocflam3 3D; and (b) CFX

Figure 10 shows the computed $\mathrm{OH}^{*}$ molar concentrations (for details on the a posteriori computation, see [7]) in comparison to the experimental $\mathrm{OH}^{*}$ emission images from Candel et al. [5]. It should be noted that the experimental data are time-averaged data, whereas the CFD results stem from the solution of time- and density-averaged equations. To further assess this, it would be interesting to compare them with simulation results from time-accurate CFD, which are, however, not available here. While in comparison with the test data both the Rocflam3 3D simulation and the axisymmetric CFX simulation reproduce the flame shape quite well, they unveil completely different flame lengths. The $\mathrm{OH}^{*}$ emission image unfortunately does not give an information about the flame length as it only covers a part of the flame. The absolute levels of the $\mathrm{OH}^{*}$ concentrations are different for the two simulations, but the experiment does not give a reference value. Thereby, $\mathrm{CFX}$ predicts a higher $\mathrm{OH}^{*}$ concentration than Rocflam3.

At this point, it is important to emphasize again that the Rocflam3 3D simulation, which has been performed on a quarter segment of the square cross section, does not show an axisymmetric flame shape (see Fig. 9) as assumed by both the $2 \mathrm{D}$ /axisymmetric simulations and the Abel transformation which has been applied to the test data in order to generate the shown $\mathrm{OH}^{*}$ emission images. This suggests that the comparison with the $\mathrm{OH}^{*}$ emission image should 


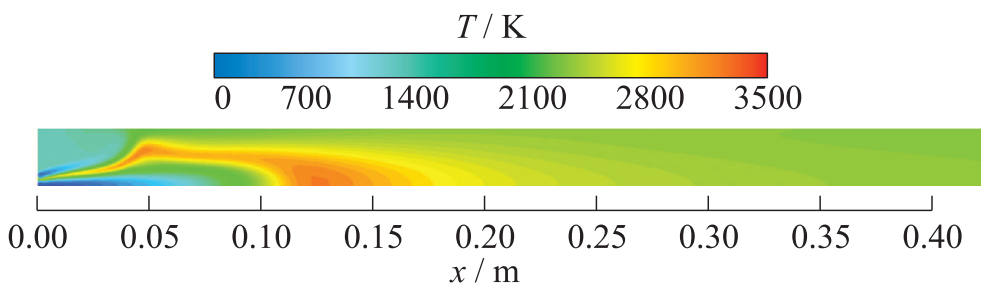

(a)

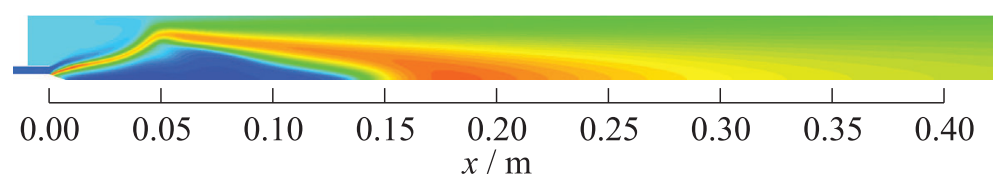

(b)

Figure 11 Temperature fields for the Mascotte A-10 test case: (a) Rocflam3 3D; and (b) CFX

not be exaggerated - a more or less good agreement with the $\mathrm{OH}^{*}$ emission image might be as much as can be concluded from this comparison.

Figure 11 shows the temperature fields from Rocflam 3 and CFX. In the recirculation region, which is located in the upper left corner of the domain, CFX computes lower temperatures than Rocflam3. Looking at the cold oxygen-rich cores, CFX shows a very long and broad cold core thereby indicating a weak mixing normal to the main flow direction. The maximum temperatures computed by Rocflam3 and CFX are 3112 and $3254 \mathrm{~K}$, respectively. Due to turbulencechemistry interaction, they are lower than the stoichiometric combustion temperature of $3380 \mathrm{~K}$ at 10 bar (computed with CEA2 (Chemical Equilibrium with Applications v.2) [32]).

In order to further assess the agreement of the simulations with the available test data, attention is now attracted to the quantitative comparison with the discrete temperature values from CARS measurements as shown in Fig. 12. Figure 12 is divided into four charts, each showing the axial temperature profiles at a distinct radial location $(0,5,10$, and $15 \mathrm{~mm})$. It should be noted that the 3D Rocflam3 simulation results are shown in the form of temperature profiles evaluated in the $x-y$-plane.

The Rocflam3 temperatures show very satisfactory agreement with the CARS data in all four charts. There are only a few measuring points where Rocflam 3 exceeds the standard deviation range around the measured mean value. The CFX results unveil too low temperature values on the axis $(y=0 \mathrm{~mm})$ in the first part of the chamber indicating that the computed cold and dense core might be too long in this simulation. However, the agreement with both Rocflam 3 and 


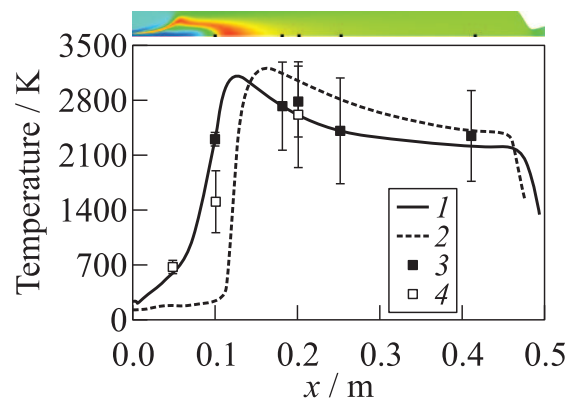

(a)

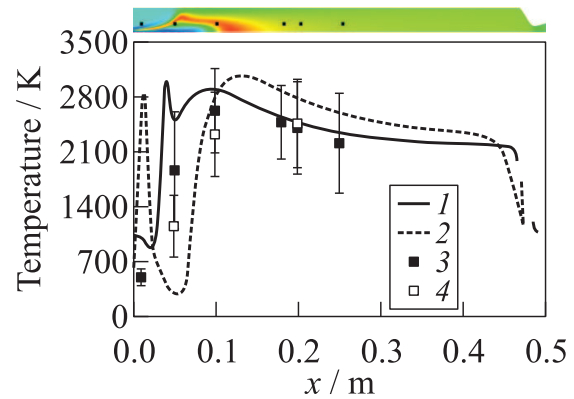

(c)

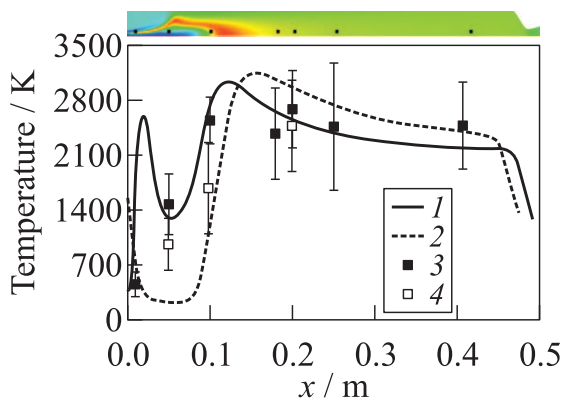

(b)

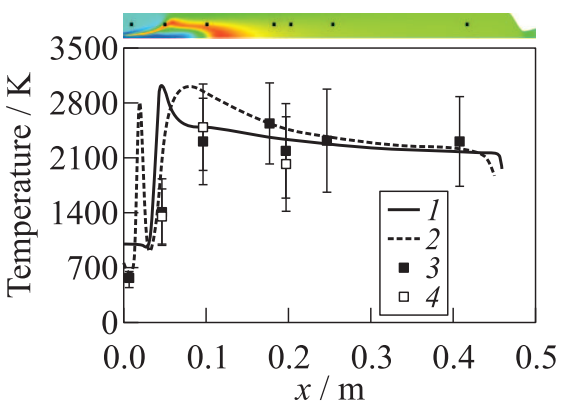

(d)

Figure 12 Temperature profiles along the $x$-axis for different radial locations $((a) y$ $=0 \mathrm{~mm} ;(b) 5 ;(c) 10 ;$ and $(d) y=15 \mathrm{~mm})$ and comparison to available CARS test data (from $[5,6]$ ); see top pictures for orientation: 1 - Rocflam3; $2-\mathrm{CFX} ; 3-$ CARS $\mathrm{H}_{2}$; and $4-$ CARS $\mathrm{H}_{2} \mathrm{O}$

the CARS data becomes better with increasing radial distance from the axis. According to the CEA2 code [32], the chamber temperature for the A-10 case is around $2118 \mathrm{~K}$, a value which is approached by both Rocflam 3 and CFX at the end of the cylindrical part.

\subsection{Mascotte A-60 Test Case}

Before comparing the simulation results from Rocflam3, TAU, and CFX for the A-60 test case, attention will again be attracted to the temperature fields computed by Rocflam3 in a $3 \mathrm{D}$ and a $2 \mathrm{D}$ /axisymmetric simulation visualized in Fig. 13. In contrast to the A-10 test case, where major differences between the $2 \mathrm{D}$ and $3 \mathrm{D}$ results are given, they are much more similar here - primarily, the positions of the flame shoulder are slightly different. The probable main reason 


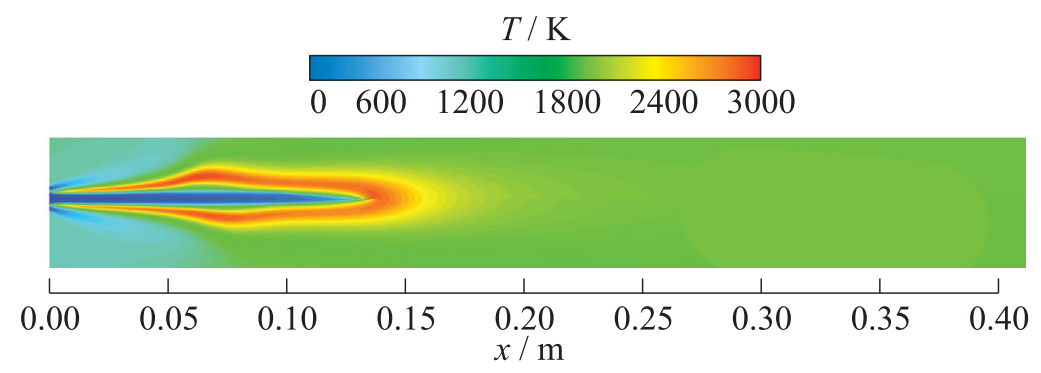

Figure 13 Temperature fields for the Mascotte A-60 test case - Rocflam3 3D (upper part) vs. $2 \mathrm{D} /$ axisymmetric (lower part)

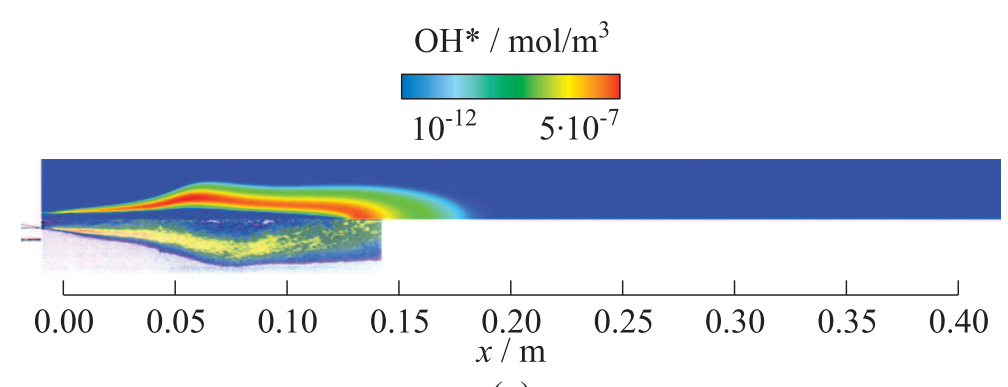

(a)

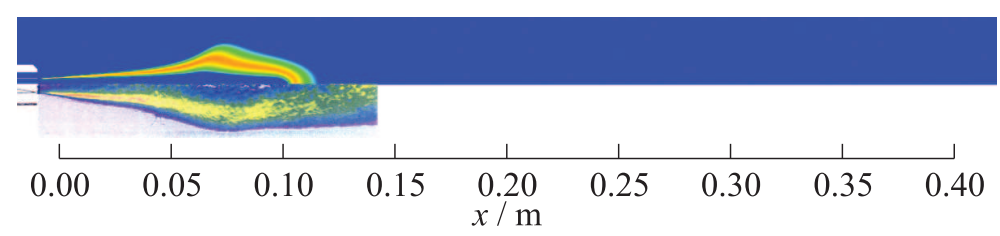

(b)

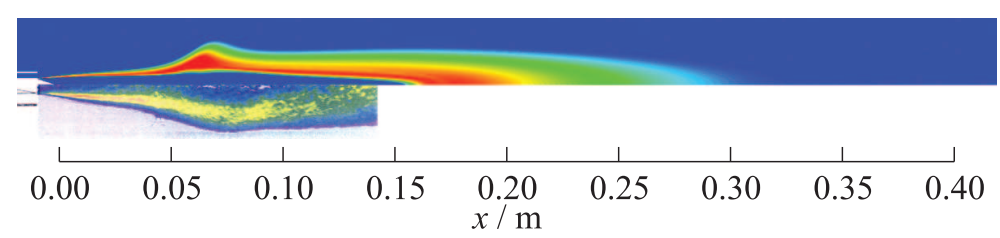

(c)

Figure $14 \mathrm{OH}^{*}$ radiation fields for the Mascotte A-60 test case (comparison between simulation results and experimental emission image obtained from [9]): (a) Rocflam3 3D; (b) TAU; and (c) CFX 
for this is that the Rocflam3 3D simulation of the A-60 test case computes a flame shape which is almost axisymmetric (not shown here). This is not the case for the A-10 test case, i.e., the axisymmetric modeling in Rocflam-II is a better approximation for the A-60 case than for the A-10 case. This also leads to the fact that the application of the Abel transformation for the processing of the $\mathrm{OH}^{*}$ emission images seems to be better suited in this case. Nevertheless, all following figures show Rocflam3 3D results.

Figure 14 shows a qualitative comparison of the numerically determined $\mathrm{OH}^{*}$ molar concentrations (for details on the applied a posteriori method, see [7]) with the experimental $\mathrm{OH}^{*}$ emission image. The emission images again give only qualitative information. The $\mathrm{OH}^{*}$ comparison shows that all three codes compute qualitatively similar flame shapes — just as for the A-10 test case: the TAU flame is the shortest and CFX produces the longest shape. As the flame is not fully captured in the measurement, it remains unclear how long it actually is just as for the A-10 test case. Nonetheless, TAU seems to underestimate the flame length somewhat. While the axial position of maximal radial flame spread ("shoulder") is captured quite well by all methods, the three simulations show $\mathrm{OH}^{*}$ concentration maxima at different locations. The experimental $\mathrm{OH}^{*}$ inten-

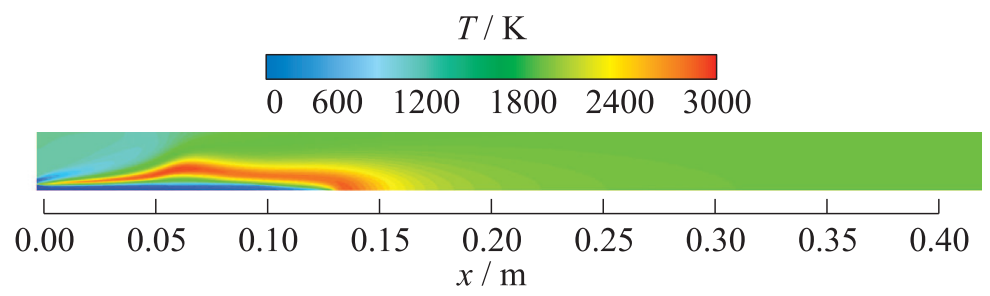

(a)

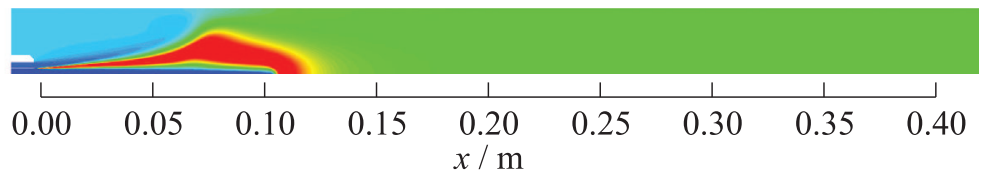

(b)

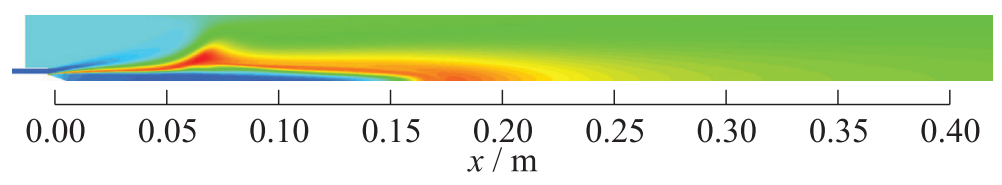

(c)

Figure 15 Computed temperature fields for the Mascotte A-60 test case: (a) Rocflam3 3D; (b) TAU; and (c) CFX 
sity is the strongest in the shear layer close to the LOx core and monotonically drops towards the flame tip. Only TAU captures this, Rocflam3 and CFX show $\mathrm{OH}^{*}$ concentration maxima in the shoulder and the flame tip.

In comparison to the A-10 test case, the A-60 case features a smaller flame opening angle and the flame shoulder is located further downstream, but the computed flame lengths are not that different. There is obviously a correlation between the propellant injection momentums (notably higher for A-60) and the flame shape, which has to be reproduced by the simulations here.

Figure 15 visualizes the temperature fields computed in the three simulations. The maximum temperature distribution follows the flame shape shown in the $\mathrm{OH}^{*}$ concentration plots in Fig. 14. Injected hydrogen and oxygen are seen as cold streams entering the chamber, the flame develops between both streams. Consistent with the classical literature, the flame is seen to isolate oxygen and hydrogen stream in all computations. TAU predicts the coldest recirculation zone but the hottest flame: the adiabatic flame temperature of stoichiometric $\mathrm{H}_{2} / \mathrm{O}_{2}$ combustion at 60 bar of approximately $3623 \mathrm{~K}$ (computed with CEA2 [32]) is slightly overestimated by TAU, whereas Rocflam3 and CFX give maximum temperatures of 2840 and $3490 \mathrm{~K}$, respectively. This discrepancy is caused by

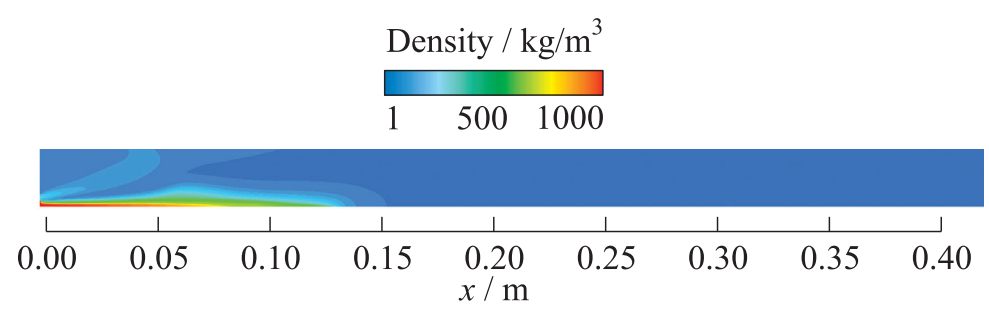

(a)

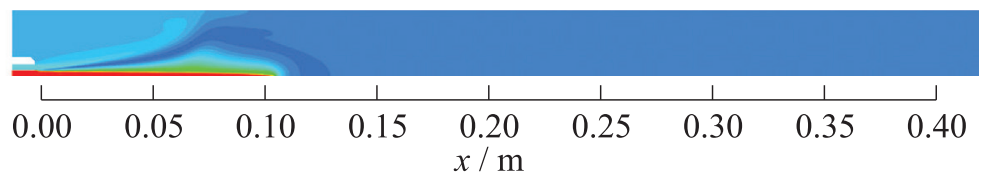

(b)

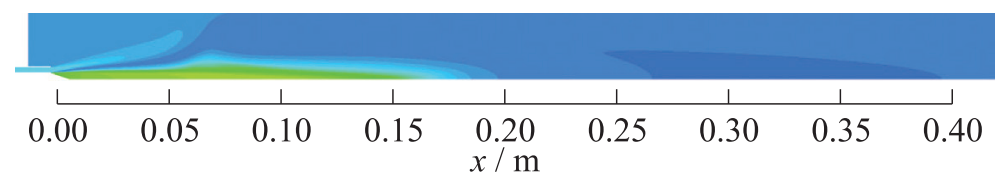

(c)

Figure 16 Density fields for the Mascotte A-60 test case (exponential color scale): (a) Rocflam3 3D; (b) TAU; and (c) CFX 
different levels of turbulence in the flame region. The difference is much stronger than in the A-10 test case. Going along with the shortest and hottest flame, the shortest cold core is in the TAU computation. The maximum flame temperature computed by Rocflam3 for the A-60 test case is lower than the value computed for the A-10 test case. This is due to the high inflow turbulence which had to be prescribed in Rocflam3 in order to reproduce the characteristic flame shape for the A-60 test case.

It is interesting to evaluate the length of the cold core when the density, visualized in Fig. 16, is taken into account as well. Rocflam 3 and TAU model the injected oxygen as an Eulerian continuum, the high LOx core density of $1100 \mathrm{~kg} / \mathrm{m}^{3}$ is, hence, reflected in the contour plot. The CFX simulation employs a Lagrangian treatment of LOx droplets, the high density is thus present in the disperse Lagrangian phase instead of the Eulerian carrier phase and is due to that not visible in Fig. 16. So, while CFX predicts the longest cold core, TAU predicts oxygen core with the highest density.

Figures 17 and 18 show a comparison of experimentally obtained temperature profiles with simulation results. Figure 17 shows radial profiles and Fig. 18 shows an axial one. All simulation results in Fig. $17 a$, which shows the radial temperature profiles at the axial position $x=50 \mathrm{~mm}$, exhibit a distinct peak close to the injector before flattening out to a plateau closer to the chamber wall. As before, TAU predicts the highest peak temperatures, whereas Rocflam 3 and CFX predict lower peak temperatures due to the influence of turbulence-chemistry interaction taken into account by these two simulations. The experimental data does not exhibit a peak at all but remains rather level, the value lying between Rocflam 3 and TAU outside of the peak. The experimental data not showing a peak at all here is misleading as the $\mathrm{OH}^{*}$ emission image clearly shows that the flame is present at the axial position $x=50 \mathrm{~mm}$ (see Fig. 14). This suggests that there must be a temperature peak which is obviously not resolved by the CARS measurements here.

At the axial position of $x=100 \mathrm{~mm}$ as shown in Fig. 17b, the three temperature profiles from the simulations again show similar general shapes while the absolute values partly differ considerably. The profiles from CFX and Rocflam3 almost coincide for a radial position farther than $5 \mathrm{~mm}$ from the centerline and agree sufficiently well with the measurements. Towards the centerline, all three codes predict a cold core, while the experimental results show a higher temperature at the axis. However, the validation rate of $5 \%$ of the CARS data is rather low here. This is probably due to the fact that the CARS data have been obtained based on $\mathrm{H}_{2}$ molecules which might not occur at the axis quite often. It should also be kept in mind that the CARS data by Habiballah et al. [10] are valid for a slightly different load point (see subsection 2.2).

Finally, Fig. 18 compares the CFD results with an axial CARS temperature profile which is - due to missing information - assumed to contain values measured on the axis. Three regions may be distinguished: a cool core at the 


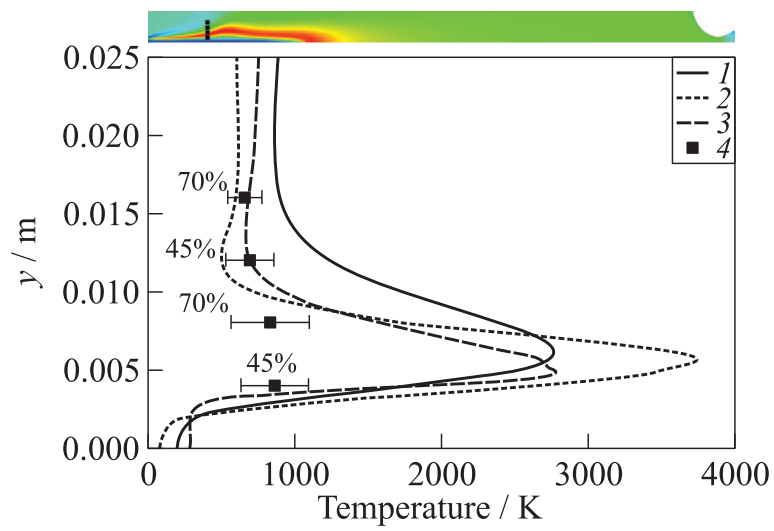

(a)

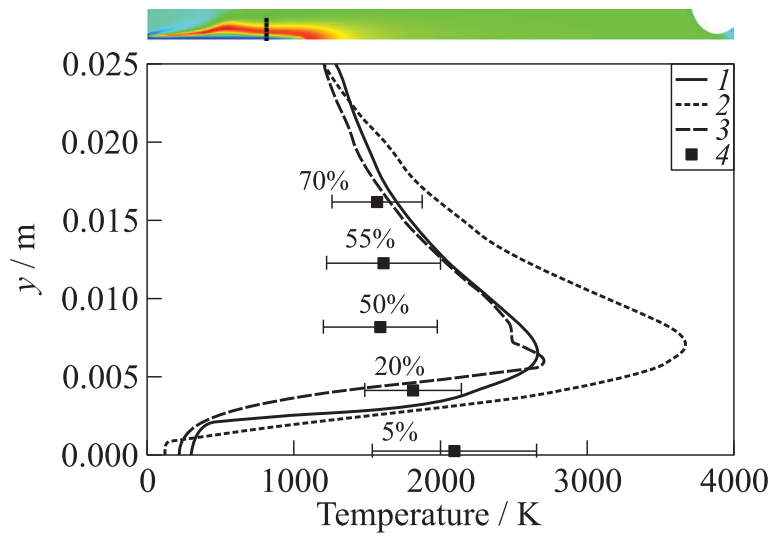

(b)

Figure 17 Temperature profiles along the $y$ axis for $x=50(a)$ and $100 \mathrm{~mm}(b)$ and comparison to available CARS test data (from [10], with validation rates); see top pictures (Rocflam3 result) for orientation: 1 - Rocflam3; 2 - TAU; $3-$ CFX; and 4 - text data

beginning, the hot flame with a peak in the center, and a plateau in the chamber behind the flame. The plateau approaches the value of $\sim 1600 \mathrm{~K}$, which is the adiabatic flame temperature that CEA2 [32] computes for this test case inside the chamber. The temperature profiles from the simulations are again similar in shape, but differ regarding absolute values and also regarding the axial position of the maximum temperature peak. Interestingly, the three codes predict different parts well: CFX captures the low temperature in the core; TAU the position and magnitude of the peak; and Rocflam3 the plateau behind 


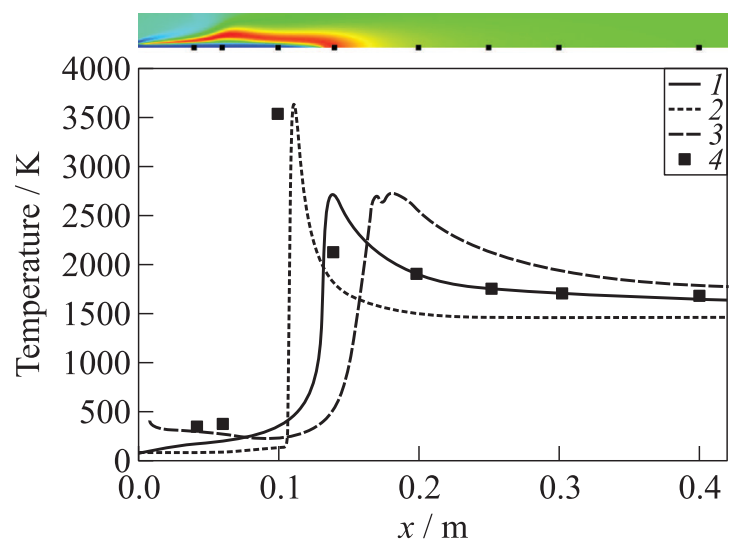

Figure 18 Temperature profiles along the $x$ axis and comparison to available test data [11]; see top picture (Rocflam3 result) for orientation: 1 - Rocflam3; 2 - TAU; 3 - CFX; and 4 - text data

the flame. The main reason why CFX shows higher temperatures in the core than Rocflam3 and TAU is that the Lagrangian approach is applied in CFX. It must be noted that Habiballah et al. [10] give a temperature of $\sim 2100 \mathrm{~K}$ for $x=100 \mathrm{~mm}$ on the center axis (see Fig. 17b) while Poschner and Pfitzner [11] give a value of $\sim 3500 \mathrm{~K}$ for the same position (see Fig. 18). This is indeed a bit confusing and cannot be finally assessed here. It stands out that the value given by Poschner and Pfitzner [11] is the only one which is close to the adiabatic flame temperature for stoichiometric combustion inside a diffusion flame at 60 bar. However, turbulence is expected to reduce the flame temperature here.

\section{CONCLUDING REMARKS}

This paper provides a comparison between the three different codes: Rocflam3, CFX, and TAU, being applied to the Mascotte A-10 and A-60 test cases, which operate in the sub- and supercritical pressure regimes of cryogenic coaxial injection.

The 3D Rocflam3 as well as the 2D/axisymmetric CFX simulation results (both Euler-Lagrange) show very good agreement for the A-10 test case both with the $\mathrm{OH}^{*}$ emission image and with the available temperature values from CARS measurements.

For the high-pressure case, it is interesting to note that the three codes, which might be considered validated when comparing experimental $\mathrm{OH}^{*}$ intensity and 
numerical $\mathrm{OH}^{*}$ molar concentration, show very distinctly different flame lengths and temperature distributions. Based on these results, one may conclude that a comparison between simulation and experiment on the basis on the $\mathrm{OH}^{*}$ emission images alone is of minor significance. This is mainly due to the facts that the emission images do not give any quantitative information, that they only cover a small section of the chamber, and that they are based on the assumption of an axisymmetric flame shape. One should definitely include the CARS measurements for comparison with discrete temperatures. However, detailed information regarding the CARS method and the data evaluation procedure must be known such that simulation results can be compared in a reasonable manner. Also, very important is to be sure about the origin and the validity of the used test data. Especially for the A-60 test case, one can find several different $\mathrm{OH}^{*}$ emission images in the literature and due to the fact that no CARS data have been presented alongside with the test case description, also, different temperature profiles have been published by different authors. Therefore, special attention is necessary here.

As already seen in previous cooperative work [2], the comparison between different CFD tools proves to be a valuable practice to review the characteristics of each modeling package. Regarding the proceeding validation of the applied modeling approaches, it must be emphasized that both Mascotte test cases are not representative for real rocket thrust chambers. Therefore, from an industrial point of view, no further efforts shall be put into the improvement of the agreement between simulation and experiment for these test cases. Instead, focus will be put on the transferability of the elaborated approaches on more relevant configurations (for more information on the Airbus DS modeling philosophy, see $[3])$.

\section{ACKNOWLEDGMENTS}

The Airbus DS part of this work was performed within the National Technology Program TARES. This program is sponsored by the German Space Agency, DLR Bonn, under contract No.50RL1210. The authors also like to thank the SFB-TR40 for the cooperation.

The research leading to the TAU results has in part been carried out within the DLR ProTAU (Propulsion in TAU) project.

\section{REFERENCES}

1. Haidn, O. J., ed. 2001. 2nd Workshop (International) on Rocket Combustion Modeling, Atomization, Combustion and Heat Transfer Proceedings. DLR Lampoldshausen, Germany. 
2. Ivancic, B., H. Riedmann, M. Frey, O. Knab, S. Karl, and K. Hannemann. 2016. Investigation of different modeling approaches for computational fluid dynamics simulation of high-pressure rocket combustors. Progress in propulsion physics. Eds. M. Calabro, L. DeLuca, S. Frolov, L. Galfetti, and O. Haidn. EUCASS advances in aerospace sciences book ser. TORUS PRESS -EDP Sciences. 8:95-116.

3. Knab, O., H. Riedmann, B. Ivancic, C. Höglauer, M. Frey, and T. Aichner. 2015, Consequences of modeling demands on numerical rocket thrust chamber flow simulation tools. Progress in propulsion physics. Eds. M. Calabro, L. DeLuca, S. Frolov, L. Galfetti, and O. Haidn. EUCASS advances in aerospace sciences book ser. TORUS PRESS -EDP Sciences. 11:317-346.

4. Vingert, L., and M. Habiballah. 2001. Presentation of test case RCM-2: Cryogenic spray combustion at 10 bar at MASCOTTE. 2nd Workshop (International) on Rocket Combustion Modeling - Atomization, Combustion and Heat Transfer Proceedings. Lampolshausen, Germany.

5. Candel, S., G. Herding, R. Snyder, P. Scouflaire, C. Rolon, L. Vingert, M. Habiballah, F. Grisch, M. Pealat, P. Bouchardy, D. Stepowski, A. Cessou, and P. Colin. 1998. Experimental investigation of shear coaxial cryogenic jet flames. J. Propul. Power 14(5):826-834.

6. Grisch, F., P. Bouchardy, and W. Clauss. 2003. CARS thermometry in high pressure rocket combustors. Aerosp. Sci. Technol. 7(4):317-330.

7. Fiala, T., and T. Sattelmayer. 2013. A posteriori computation of $\mathrm{OH}^{*}$ radiation from numerical simulations in rocket combustion chambers. 5th European Conference for Aeronautics and Space Sciences. Munich, Germany.

8. Thomas, J. L., and S. Zurbach. 2001. Presentation of test case RCM-3: Supercritical spray combustion at 60 bar at MASCOTTE. 2nd Workshop (International) on Rocket Combustion Modeling - Atomization, Combustion and Heat Transfer Proceedings. Lampoldshausen, Germany.

9. Cheng, G., and R. Farmer. 2002. CFD spray combustion model for liquid rocket engine injector analyses. 40th AIAA Aerospace Sciences Meeting and Exhibit.

10. Habiballah, M., M. Orain, F. Grisch, L. Vingert, and P. Gicquel. 2006. Experimental studies of high-pressure crygenic flames on the MASCOTTE facility. Combust. Sci. Technol. 178(1-3):101-128.

11. Poschner, M. M., and M. Pfitzner. 2008. Real gas CFD simulation of supercritical $\mathrm{H}_{2}-\mathrm{LOx}$ combustion in the Mascotte single-injector combustor using a commercial CFD code. 46th AIAA Aerospace Sciences Meeting and Exhibit. Reno, NV.

12. Patankar, S. V., and D. B. Spalding. 1972. A calculation procedure for heat, mass and momentum transfer in three-dimensional parabolic flows. Int. J. Heat Mass Tran. 15(10):1787-1806.

13. Jones, W. P., and B. E. Launder. 1972. The prediction of laminarization with a twoequation model of turbulence. Int. J. Heat Mass Tran. 15:301-314. 
14. Kniesner, B., M. Frey, and O. Knab. 2011. Numerical investigation of gas generator and preburner flows for rocket engine applications. EUCASS. St. Petersburg, Russia.

15. Abramzon, B., and W. A. Sirignano. 1988. Droplet vaporization model for spray combustion calculations. AIAA 26th Aerospace Sciences Meeting. Reno, NV.

16. Riedmann, H., B. Kniesner, M. Frey, and C.-D. Munz. 2014. 3D modeling of spray combustion and flow in a $40 \mathrm{kN} \mathrm{H} / \mathrm{O}_{2}$ subscale rocket thrust chamber. Space Propulsion Conference. Cologne, Germany.

17. Gerhold, T. 2005. Overview of the hybrid RANS code TAU MEGAFLOW - numerical flow simulation for aircraft design. Notes on numerical fluid mechanics and multidisciplinary design. Springer. 89:81-92.

18. Schwamborn, D., T. Gerhold, and R. Heinrich. 2006. The DLR TAU-code: Recent applications in research and industry. European Conference in Computational Fluid Dynamics. Egmond an Zee, Netherlands.

19. Spallart, P.R., and S. R. Allmaras. 1992. A one-equation turbulence model for aerodynamic flows. AIAA Paper No. 92-0439.

20. Jachimowski, C.. 1988. An analytical study of the hydrogen-air reaction mechanism with application to scramjet combustion. NASA Technical Paper No. 2791.

21. Lempke, M., P. Gerlinger, and M. Aigner. 2013. Assumed PDF modeling in rocket combustor simulations. Progress in propulsion physics. Eds. L. T. DeLuca, C. Bonnal, O. Haidn, and S. M. Frolov. EUCASS advances in aerospace sciences book ser. TORUS PRESS-EDP Sciences. 4:569-582.

22. Lacaze, G., and J. C. Oefelein. 2012. A non-premixed combustion model based on flame structure analysis at supercritical pressures. Combust. Flame 159:2087-2103.

23. Baniti, D. T., M. Raju, P. C. Ma, M. Ihme, and J.-P. Hickey. 2017. Seven questions about supercritical fluids - towards a new fluid state diagram. AIAA Paper No. 2017-1106.

24. Mayer, W., and H. Tamura. 1996. Propellant injection in a liquid oxygen / gaseous hydrogen rocket engine. J. Propul. Power 12(6):1137-1147.

25. Younglove, B. A. 1982. Thermophysical properties of fluids. I. Argon, ethylene, parahydrogen, nitrogen, nitrogen trifluoride, and oxygen. J. Phys. Chem. Ref. Data 11(suppl. 1). 356 p.

26. Lemmon, E. W., and R. T. Jacobsen. 2004. Viscosity and thermal conductivity equations for nitrogen, oxygen, argon, and air. Int. J. Thermophys. 21-69.

27. Banuti, D. T., and K. Hannemann. 2014. Efficient multiphase rocket propellant injection model with high quality equation of state. 4th Space Propulsion Conference Proceedings. Cologne, Germany.

28. Banuti, D. T., and K. Hannemann. 2014. Application of a real-gaslibrary multi-fluid-mixing model to supercritical single injector flow. 50th AIAA/ASME/SAE/ASEE Joint Propulsion Conference Proceedings. Cleveland, $\mathrm{OH}$. 
29. Banuti, D. T., V. Hannemann, K. Hannemann, and B. Weigand. 2016. An efficient multi-fluid-mixing model for real gas reacting flows in liquid propellant rocket emgines. Combust. Flame 168:98-112.

30. Menter, F., J. Carregal Ferreira, T. Esch, and B. Konno. 2003. The SST turbulence model with improved wall treatment for heat transfer predictions in gas turbines. Gas Turbine Congress (International) Proceedings. Tokyo, Japan.

31. Ivancic, B., M. Frey, and O. Knab. 2010. 3D-numerical investigation of turbulent combustion and heat transfer processes in $\mathrm{H}_{2}-\mathrm{O}_{2}$ liquid rocket combustors. Space Propulsion Conference. San Sebastian, Spain.

32. McBride, B. J., and S. Gordon. 1996. Computer program for calculation of complex chemical equilibrium compositions and applications. Cleveland, OH. NASA Reference Publication 1311. 